\title{
Mass generation via the Higgs boson and the quark condensate of the QCD vacuum
}

\author{
Martin Schumacher* \\ II. Physikalisches Institut der Universität Göttingen, Friedrich-Hund-Platz 1 \\ D-37077 Göttingen, Germany
}

\begin{abstract}
The Higgs boson, recently discovered with a mass of $125.09 \pm 0.24 \mathrm{GeV}$ is known to mediate the masses of elementary particles, but only $2 \%$ of the mass of the nucleon. Extending a previous investigation [1] and including the strange-quark sector, hadron masses are derived from the quark condensate of the QCD vacuum and from the effects of the Higgs boson. These calculations include the $\pi$ meson, the nucleon and the scalar mesons $\sigma(600), \kappa(800), a_{0}(980), f_{0}(980)$ and $f_{0}(1370)$. The predicted second $\sigma$ meson, $\sigma^{\prime}(1344)=|s \bar{s}\rangle$, is investigated and identified with the $f_{0}(1370)$ meson. An outlook is given on the hyperons $\Lambda, \Sigma^{0, \pm}$ and $\Xi^{0,-}$.
\end{abstract}

\section{Introduction}

In the standard model the masses of elementary particles arise from the Higgs field acting on the originally massless particles. When applied to the visible matter of the universe this explanation remains unsatisfactory as long as we consider the vacuum as an empty space. The QCD vacuum contains a condensate of up and down quarks. Condensate means that the $q \bar{q}$ pairs are correlated via inter-quark forces mediated by gluon exchanges. As part of the vacuum structure the $q \bar{q}$ pairs have to be in a scalar-isosclar configuration. This suggests that the vacuum condensate may be described in terms of a scalar-isoscalar particle, $|\sigma\rangle=(|u \bar{u}\rangle+|d \bar{d}\rangle) / \sqrt{2}$, providing the $\sigma$ field. These two descriptions in terms of a vacuum condensate or a $\sigma$ field are essentially equivalent and are the bases of the Nambu-Jona-Lasinio (NJL) model [2,8] and the linear $\sigma$ model (L $\sigma \mathrm{M})$, , 9] respectively. Furthermore, it is possible write down a bosonized version of the NJL model where the vacuum condensate is replaced by the vacuum expectation value of the $\sigma$ field.

In the QCD vacuum the largest part of the mass $M$ of an originally massless quark, up $(\mathrm{u})$ or down $(\mathrm{d})$, is generated independent of the presence of the Higgs field and amounts to $M=326 \mathrm{MeV}$ [1]. The Higgs field only adds a small additional part to the total constituent-quark mass leading to $m_{u}=331 \mathrm{MeV}$ and $m_{d}=335 \mathrm{MeV}$ for the up and down quark, respectively [1]. These constituent quarks are the building blocks of the nucleon in a similar way as the nucleons are in case of nuclei. Quantitatively, we obtain the experimental masses of the nucleons after including a binding energy of $19.6 \mathrm{MeV}$ and $20.5 \mathrm{MeV}$ per constituent quark for the proton and neutron, respectively, again in analogy to the nuclear case where the binding energies are $2.83 \mathrm{MeV}$ per nucleon for ${ }_{1}^{3} \mathrm{H}$ and $2.57 \mathrm{MeV}$ per nucleon for ${ }_{2}^{3} \mathrm{He}$.

In the present work we extend our previous [1 investigation by exploring in more detail the rules according to which the effects of electroweak (EW) and strong-interaction

*mschuma3@gwdg.de 
symmetry breaking combine in order to generate the masses of hadrons. As a test of the concept, the mass of the $\pi$ meson is precisely predicted on an absolute scale. In the strange-quark sector the Higgs boson is responsible for about $1 / 3$ of the constituentquark mass, so that effects of the interplay of the two components of mass generation become essential. Progress is made by taking into account the predicted second $\sigma$ meson, $\sigma^{\prime}(1344)=|s \bar{s}\rangle$ [7]. It is found that the coupling constant of the $s$-quark coupling to the $\sigma^{\prime}$ meson is larger than the corresponding quantity of the $u$ and $d$ quarks coupling to the $\sigma$ meson by a factor of $\sqrt{2}$. This leads to a considerable increase of the constituentquark masses in the strange-quark sector in comparison with the ones in the non-strange sector already in the chiral limit, i.e. without the effects of the Higgs boson. There is an additional sizable increase of the mass generation mediated by the Higgs boson due to a $\sim 20$ times stronger coupling of the $s$ quark to the Higgs boson in comparison to the $u$ and $d$ quarks.

In addition to the progress made in [1] as described above that paper contains a History of the subject from Schwinger's seminal work of 1957 [10] to the discovery of the BroutEnglert-Higgs (BEH) mechnism, with emphasis on the Nobel prize awarded to Nambu in 2008. This is the reason why paper [1] has been published as a supplement of the Nobel lectures of Englert [11] and Higgs [12].

\section{Symmetry breaking in the non-strange sector}

In Figure 1 the symmetry breaking process is illustrated. The left panel corresponds to the $\mathrm{L} \sigma \mathrm{M}$, the right panel to the bosonized NJL model, together with their EW counterparts. In the left panel symmetry breaking provides us with a vacuum expectation $v_{H}$ of the Higgs field $\mathrm{H}$ and $v_{\sigma}$ of the $\sigma$-field. Without the effects of the Higgs field the stronginteraction Nambu-Goldstone bosons, $\pi$, are massless. The $\pi$ mesons generate mass via the interaction with the Higgs field in the presence of the QCD quark condensate, as will be outlined below. The EW counterparts of the $\pi$ mesons are the longitudinal components $W_{l}$ of the weak vector bosons $W$. These longitudinal components are transferred into the originally massless weak vector bosons $\mathrm{W}$ via the Brout-Englert-Higgs (BEH) mechanism. In the right panel the view of the bosonized NJL model is presented. The originally massless quarks interact via the exchanges of a $\sigma$ meson or a Higgs boson with the respective $\sigma$ field of the QCD vacuum or the Higgs field of the EW vacuum. As long as we consider the two symmetry breaking processes separately we can write down [1]

$$
\begin{aligned}
& M=g v_{\sigma}^{\mathrm{cl}}, \\
& m_{u}^{0}=2.03 \times 10^{-5} v_{H}, \\
& m_{d}^{0}=3.66 \times 10^{-5} v_{H} .
\end{aligned}
$$

The quantity $g$ is the quark- $\sigma$ coupling constant which has been derived to be $g=\frac{2 \pi}{\sqrt{3}}$. This quantity leads via Eq. (11) to the constituent-quark mass $M=326 \mathrm{MeV}$ in the chiral limit $(\mathrm{cl})$, i.e. without the effects of the Higgs boson. The quantity $v_{\sigma}^{\mathrm{cl}} \equiv f_{\pi}^{\mathrm{cl}}=89.8 \mathrm{MeV}$ is the pion decay constant in the chiral limit, serving as vacuum expectation value $\left(v_{\sigma}\right)$. The quantity $v_{H}=246 \mathrm{GeV}$ is the vacuum expectation value of the Higgs field, leading to the current-quark masses $m_{u}^{0}=5 \mathrm{MeV}$ and $m_{d}^{0}=9 \mathrm{MeV}$. These values are the well known 


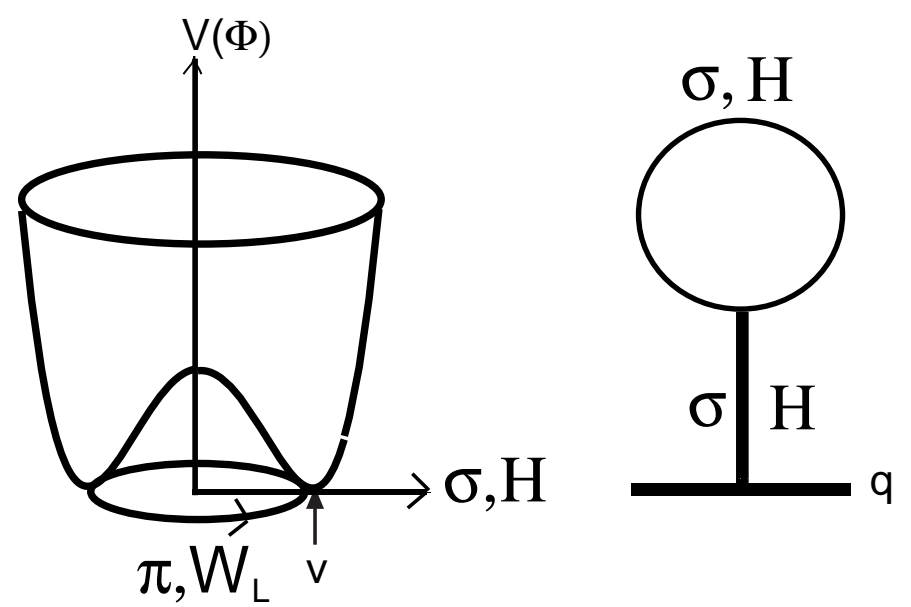

Figure 1: Strong-interaction and EW interaction symmetry breaking. Left panel: The L $\sigma \mathrm{M}$ together with the EW counterpart. Right panel: The bosonized NJL model together with the EW counterpart.

current-quark masses entering into low-energy QCD via explicit symmetry breaking. The coupling constants in Eqs. (2) and (3) are chosen such that these values are reproduced.

In the following we study the laws according to which the two sources of mass generation combine in order to generate the observable particle masses. For this purpose we write down the well known NJL equation and refer to [1] for more details

$$
\mathcal{L}_{\mathrm{NJL}}=\bar{\psi}\left(i \not \partial-m_{0}\right) \psi+\frac{G}{2}\left[(\bar{\psi} \psi)^{2}+\left(\bar{\psi} i \gamma_{5} \boldsymbol{\tau} \psi\right)^{2}\right] .
$$

In Eq. (4) the interaction between fermions is parameterized by the four-fermion interaction constant G. Explicit symmetry breaking as mediated by the Higgs boson is represented by the trace of the current-quark mass matrix $m_{0}$ [5]. From Eq. (44) the constituent-quark mass in the chiral limit (cl) may be derived via the relation

$$
M=G|\langle\bar{\psi} \psi\rangle|^{\mathrm{cl}} \text {. }
$$

The bosonization of Eq. (4) is obtained by replacing $\mathrm{G}$ by a propagator

$$
G \rightarrow \frac{g^{2}}{\left(m_{\sigma}^{\mathrm{cl}}\right)^{2}-q^{2}}, \quad q^{2} \rightarrow 0
$$

where $m_{\sigma}^{\mathrm{cl}}$ is the mass of the $\sigma$ meson in the chiral limit and $q$ the momentum carried by the $\sigma$ meson, and by introducing the $\sigma$ and $\pi$ fields via

$$
\sigma=-\frac{G}{g} \bar{\psi} \psi, \quad \boldsymbol{\pi}=-\frac{G}{g} \bar{\psi} i \gamma_{5} \boldsymbol{\tau} \psi
$$

Using the Nambu relation $m_{\sigma}^{c l}=2 M$, the quark- $\sigma$ coupling constant $g=\frac{2 \pi}{\sqrt{3}}, M=g f_{\pi}^{\mathrm{cl}}$ and $f_{\pi}^{\mathrm{cl}}=89.8 \mathrm{MeV}$ we arrive at

$$
|\langle\bar{\psi} \psi\rangle|^{\mathrm{cl}}=\frac{8 \pi}{\sqrt{3}}\left(f_{\pi}^{\mathrm{cl}}\right)^{3}=(219 \mathrm{MeV})^{3}, \quad G=\frac{1}{4\left(f_{\pi}^{\mathrm{cl}}\right)^{2}}=3.10 \times 10^{-5} \mathrm{MeV}^{-2} .
$$


In the present case of small current-quark masses it is straightforward to arrive at a version which includes the effects of the Higgs boson by replacing $f_{\pi}^{\mathrm{cl}}$ with $f_{\pi}$. The result is given by

$$
|\langle\bar{\psi} \psi\rangle|=\frac{8 \pi}{\sqrt{3}}\left(f_{\pi}\right)^{3}=(225 \mathrm{MeV})^{3}, \quad f_{\pi}=(92.43 \pm 0.26) \mathrm{MeV} .
$$

This equation shows that a representation of the QCD quark condensate through the vacuum expectation value of the $\sigma$ field is possible and leads to a prediction for a value of the vacuum condensate which is tested and found valid in the next subsection.

Fig. 1 suggests a formal similarity of strong-interaction and EW interaction symmetry breaking. This formal similarity is incomplete because at the present state of our knowledge we have to consider the Higgs boson as elementary, i.e. without a fermion-antifermion substructure. Formerly, substructures of the Higgs field in terms of techniquark-antitechniquark pairs or top-antitop quark pairs have been discussed. In the techniquark-model strong self-coupling has been discussed leading to a predicted Higgs-boson mass of $\sim 1$ $\mathrm{TeV}$. In the top-quark model the predicted Higgs boson mass is expected to be $\sim 2 m_{t}$. With a comparatively small experimental mass of $m_{H}=125.1 \mathrm{GeV}$ of the Higgs boson these models seem to be excluded. In case of the left panel we may write down a potential in the form 13

$$
V(\phi)=-\mu^{2} \phi^{\dagger} \phi+\lambda\left(\phi^{\dagger} \phi\right)^{2}, \quad \mu^{2}>0, \quad \lambda>0 .
$$

The potential $V(\phi)$ then has its minimum at a finite value of $|\phi|$ where

$$
\phi^{\dagger} \phi=\frac{1}{2}\left(\phi_{1}^{2}+\phi_{2}^{2}+\phi_{3}^{2}+\phi_{4}^{2}\right)=\frac{\mu^{2}}{2 \lambda} .
$$

We can choose, say,

$$
\phi_{1}=\phi_{2}=\phi_{4}=0, \quad\left\langle\phi_{3}\right\rangle=\sqrt{\frac{\mu^{2}}{\lambda}} \equiv v_{H}
$$

where $v_{H}=246 \mathrm{GeV}$ is the vacuum expectation value of the Higgs field. In the case of strong interaction the corresponding relations are

$$
\phi^{\dagger} \phi=\frac{1}{2}\left(\sigma^{2}+\pi^{2}\right)=\frac{\mu^{2}}{2 \lambda}
$$

with

$$
\boldsymbol{\pi}=0, \quad\langle\sigma\rangle=\sqrt{\frac{\mu^{2}}{\lambda}} \equiv v_{\sigma}^{\mathrm{cl}} \equiv f_{\pi}^{\mathrm{cl}}
$$

It is of interest to compare the selfcoupling strengths of strong interaction symmetry breaking with the one of EW symmetry breaking. The sigma-meson mass in the chiral limit may be expressed in two ways [1]

$$
m_{\sigma}^{c l}=2 g v_{\sigma}^{\mathrm{cl}}, \quad m_{\sigma}^{\mathrm{cl}}=\sqrt{2 \lambda_{\sigma}} v_{\sigma}^{\mathrm{cl}}
$$

where the first version corresponds to the NJL model and the second to the L $\sigma \mathrm{M}$. With $v_{\sigma}=f_{\sigma}^{c l}=89.8 \mathrm{MeV}$ and $g=\frac{2 \pi}{\sqrt{3}}=3.63$ this leads to

$$
m_{\sigma}^{\mathrm{cl}}=652 \mathrm{MeV} \text { and } \lambda_{\sigma}=\frac{8 \pi^{2}}{3}=26.3 .
$$


For the Higgs boson we have

$$
m_{H}=\sqrt{2 \lambda_{H}} v_{H}, m_{H}=125.1 \mathrm{GeV} \text { and } v_{H}=246 \mathrm{GeV}
$$

leading to

$$
\lambda_{H}=0.130 .
$$

We see that the strong-interaction selfcoupling is a factor $\lambda_{\sigma} / \lambda_{H}=202$ larger than the EW selfcoupling. But except for this, there indeed is a formal similarity between the two versions of symmetry breaking. This may help to get a better understanding of the underlying physics of the Higgs boson.

\subsection{Prediction of the masses of the $\pi$ and the $\sigma$ meson}

The mass of the $\pi$ meson is given by the Gell-Mann-Oakes-Renner (GOR) relation in the form

$$
m_{\pi}^{2} f_{\pi}^{2}=\left(m_{u}^{0}+m_{d}^{0}\right)|\langle\bar{\psi} \psi\rangle|, \quad|\langle\bar{\psi} \psi\rangle|=\frac{1}{2}|\langle\bar{u} u+\bar{d} d\rangle|=\frac{8 \pi}{\sqrt{3}}\left(f_{\pi}\right)^{3},
$$

(see Eq. (9) leading to

$$
m_{\pi}^{2}=\left(m_{u}^{0}+m_{d}^{0}\right) \frac{8 \pi}{\sqrt{3}} f_{\pi}
$$

The numerical value derived from Eq. (20) is $m_{\pi}=137.0 \mathrm{MeV}$, to be compared with the experimental values $m_{\pi}^{0}=135.0 \mathrm{MeV}$ and $m_{\pi}^{ \pm}=139.6 \mathrm{MeV}$. Apparently, the predicted value of the $\pi$ meson mass is quite satisfactory when use is made of the current-quark masses following from Eqs. (2) and (3). Furthermore, in the neutral $\pi^{0}$ we find a Coulomb attraction between the quark and the antiquark leading to an experimental value smaller than the predicted value, whereas in the charged $\pi^{ \pm}$we find a Coulomb repulsion between the quark and the antiquark leading to an experimental value larger than the predicted value.

From Eqs. (1) - (3) we can see that the constituent quark in the chiral limit and the current quarks generate masses independently. But these masses are not the ones observed in low-energy QCD. The procedure to arrive at predictions for the observable masses has been derived from arguments given by the $\mathrm{L} \sigma \mathrm{M}$ as well as NJL models. First we calculate the mass of the $\sigma$ meson in the chiral limit via the Nambu relation

$$
m_{\sigma}^{\mathrm{cl}}=2 M=652 \mathrm{MeV} \quad \text { with } \quad M=\frac{2 \pi}{\sqrt{3}} f_{\pi}^{\mathrm{cl}}=326 \mathrm{MeV}
$$

and then use the relation

$$
m_{\sigma}=\left(4 M^{2}+\hat{m}_{\pi}^{2}\right)^{1 / 2}=666 \mathrm{MeV} .
$$

In Eq. (22) the effects of the Higgs boson enter via the average pion mass $\hat{m}_{\pi}$. It is interesting to note that in the limit of small current-quark masses the result of Eq. (22) can also be derived by simply adding the contributions from the QCD quark-condensate and from the Higgs boson, leading to

$$
\begin{aligned}
& m_{u}=M+m_{u}^{0}=331 \mathrm{MeV} \\
& m_{d}=M+m_{d}^{0}=335 \mathrm{MeV} \\
& m_{\sigma}=m_{\sigma}^{\mathrm{cl}}+m_{u}^{0}+m_{d}^{0}=666 \mathrm{MeV} .
\end{aligned}
$$


The arguments leading to an equivalence of Eqs. (22) and (25) are as follows. Eq. (22) can be written is the form

$$
m_{\sigma}=m_{\sigma}^{\mathrm{cl}}+\frac{1}{2} \frac{\hat{m}_{\pi}^{2}}{m_{\sigma}^{\mathrm{cl}}}+\cdots=m_{\sigma}^{\mathrm{cl}}+\left(m_{u}^{0}+m_{d}^{0}\right) .
$$

Here use has been made of Eqs. (20) and (21) to show that the term $\frac{1}{2} \frac{\hat{m}_{\pi}^{2}}{m_{\sigma}^{\text {cl }}}$ of Eq. (26) is equal to $\left(m_{u}^{0}+m_{d}^{0}\right)$ if higher order terms amounting to $4 \%$ and the deviation of $f_{\pi}^{\text {cl }} / f_{\pi}$ from 1 amounting to $3 \%$ are neglected.

The result shown in Eq. (25) is in line with the expectation that the $\sigma$ meson is a loosely bound object where no additional term, viz. the binding energy $B$, has to be taken in account. This is different in the case of baryons as we will see later.

\section{The fundamental structure constants magnetic mo- ment, polarizability and mass of the nucleon}

In the previous paper [1] it has been shown that it is possible to make precise predictions on an absolute scale not only for the mass of the nucleon but also for such sophisticated structure constants as there are the magnetic moment and the polarizability. This is an important finding because it implies that the underlying models are confirmed in different and complementary ways. In the present article we return to this problem and make some necessary amendments.

\subsection{The magnetic moment of the nucleon}

The magnetic moments of the nucleon are given by

$$
\begin{aligned}
\mu_{p} & =\frac{4}{3} \mu_{u}-\frac{1}{3} \mu_{d}, \\
\mu_{n} & =\frac{4}{3} \mu_{d}-\frac{1}{3} \mu_{u}
\end{aligned}
$$

in units of the nuclear magneton $\mu_{N}=e \hbar / 2 m_{p}$. Constituent-quark masses enter through the relations

$$
\mu_{u}=\frac{2}{3} \frac{m_{p}}{m_{u}}, \quad \mu_{d}=-\frac{1}{3} \frac{m_{p}}{m_{d}},
$$

where $m_{u}=331 \mathrm{MeV}$ and $m_{d}=335 \mathrm{MeV}$. This leads to the magnetic moments of the constituent quarks

$$
\mu_{u}=1.890, \quad \mu_{d}=-0.934
$$

and to predicted magnetic moments of the nucleon

$$
\mu_{p}^{\text {theor }}=2.831 \quad \mu_{n}^{\text {theor }}=-1.875 .
$$

Comparing these values with the experimental magnetic moments of the nucleon

$$
\mu_{p}^{\exp }=2.79285, \quad \mu_{n}^{\exp }=-1.91304
$$


we arrive at very small differences $\Delta \mu=\mu^{\exp }-\mu^{\text {theor }}$

$$
\Delta \mu_{p}=-0.038, \quad \Delta \mu_{n}=-0.038 \text {. }
$$

Apparently, the necessary corrections to the quark-model predictions of the magnetic moments for the proton and neutron are the same. This may help to find an explanation for these corrections. The most probable explanation may be found in terms of meson exchange currents though available calculations lead to too large values.

In the present work we are mainly interested in the small sizes of $1.4 \%-2.0 \%$ of the differences showing that the predictions obtained on the basis of the NJL model are very precise.

\subsection{The polarizabilities of the nucleon}

A nucleon in an electric field $\mathbf{E}$ and magnetic field $\mathbf{H}$ obtains an electric dipole moment $\mathbf{d}$ and magnetic dipole moment $\mathbf{m}$ given by

$$
\begin{aligned}
& \mathbf{d}=4 \pi \alpha \mathbf{E} \\
& \mathbf{m}=4 \pi \beta \mathbf{H}
\end{aligned}
$$

in a unit system where the electric charge $e$ is given by $e^{2} / 4 \pi=\alpha_{\mathrm{em}}=1 / 137.04$. The quantities $\alpha$ and $\beta$ are the electric and magnetic polarizabilities belonging to the fundamental structure constants of the nucleon. It is of importance that these quantities are composed of two components

$$
\begin{aligned}
& \alpha=\alpha^{s}+\alpha^{t}, \\
& \beta=\beta^{s}+\beta^{t}
\end{aligned}
$$

where the superscript $s$ denotes the $s$-channel contribution and the superscript $t$ the $t$ channel contribution. The $s$-channel contribution is related to the meson-photoproduction amplitudes of the nucleon via the optical theorem whereas the $t$-channel contribution is related to the $\sigma$ meson as part of the constituent-quark structure. Therefore, it is possible to use the polarizabilities as a tool to test the predicted mass and structure of the $\sigma$ meson. This is summarized in the following equations [14]

$$
\begin{aligned}
& |\sigma\rangle=\frac{1}{\sqrt{2}}(|u \bar{u}\rangle+|d \bar{d}\rangle), \quad \mathcal{M}(\sigma \rightarrow \gamma \gamma)=\frac{\alpha_{\mathrm{em}} N_{c}}{\pi f_{\pi}}\left[\left(\frac{2}{3}\right)^{2}+\left(\frac{-1}{3}\right)^{2}\right], \\
& (\alpha-\beta)_{p, n}^{t}=\frac{g_{\sigma N N} \mathcal{M}(\sigma \rightarrow \gamma \gamma)}{2 \pi m_{\sigma}^{2}}=15.2, \quad(\alpha+\beta)_{p, n}^{t}=0 \\
& \alpha_{p, n}^{t}=+7.6 \\
& \alpha_{p}^{s}=+4.5, \quad \alpha_{n, n}^{t}-7.6
\end{aligned}
$$

in units of $10^{-4} \mathrm{fm}^{3}$, where use is made of $g_{\pi N N}=g_{\sigma N N}=13.169 \pm 0.057$ and $m_{\sigma}=666$ $\mathrm{MeV}$ as predicted by the NJL model.

The polarizability components listed in Eq. (40) correspond to the $t$-channel and have been calculated from Eqs. (38) and (39). The polarizability components in Eq. 
Table 1: Total predicted polarizabilities and experimental results (unit $10^{-4} \mathrm{fm}^{3}$ )

\begin{tabular}{l|ll|ll}
\hline & $\alpha_{p}$ & $\beta_{p}$ & $\alpha_{n}$ & $\beta_{n}$ \\
\hline total predicted & +12.1 & +1.8 & +12.7 & +2.5 \\
experim. result & $+(12.0 \pm 0.6)$ & $+(1.9 \mp 0.6)$ & $+(12.5 \pm 1.7)$ & $+(2.7 \mp 1.8)$ \\
\hline
\end{tabular}

(41) correspond to the $s$-channel and have been calculated from high-precision meson photoproduction amplitudes [14].

The purpose of this subsection is to show that the $q \bar{q}$ structure of the $\sigma$ meson as given in Eq. (38) together with the mass $m_{\sigma}=666 \mathrm{MeV}$ leads to an excellent agreement of the predicted with the experimental polarizabilities of the nucleon as shown in Table 1 . Furthermore, there is a Compton-scattering experiment on the proton where the $\sigma$ meson as part of the constituent-quark structure is directly visible in the differential cross section for Compton scattering in the energy range from 400-700 MeV and at large scattering angles [15-17]. This latter experiment leads to a $\sigma$ meson mass of $m_{\sigma}=600 \pm 70 \mathrm{MeV}$ [14] in good agreement with the standard value $m_{\sigma}=666 \mathrm{MeV}$.

\subsection{The masses of the nucleons}

As shown above, the constituent-quark masses including the effects of the Higgs boson are

$$
m_{u}=331 \mathrm{MeV} \text { and } m_{d}=335 \mathrm{MeV} .
$$

This leads to the nucleon masses

$$
\begin{aligned}
& m_{p}^{0}=2 m_{u}+m_{d}=997 \mathrm{MeV}, \\
& m_{n}^{0}=2 m_{d}+m_{u}=1001 \mathrm{MeV} .
\end{aligned}
$$

The difference of these quantities from the experimental values

$$
\begin{aligned}
& m_{p}=938.27 \mathrm{MeV}, \\
& m_{n}=939.57 \mathrm{MeV},
\end{aligned}
$$

may be interpreted in terms of a binding energy $B$, leading to

$$
\begin{aligned}
& B_{p}=m_{p}^{0}-m_{p}=59 \mathrm{MeV}, \\
& B_{n}=m_{n}^{0}-m_{n}=61 \mathrm{MeV} .
\end{aligned}
$$

The larger binding energy $B_{n}$ of the neutron compared to $B_{p}$ of the proton, $B_{n}-B_{p} \approx 2$ $\mathrm{MeV}$, has previously [1] been interpreted in terms of a Coulomb attraction, which leads to zero in case of the proton but to a nonzero value of the right order of magnitude in case of the neutron. The arguments were as follows. The electromagnetic potential acting between three constituent quarks may be written in the form

$$
U=\sum_{i<j} \frac{e_{i} e_{j}}{r_{i j}} \alpha_{e m} \hbar c
$$


where the denominator has been replaced by an educated guess for the average interquark distance [1], viz. $\left\langle r_{i j}\right\rangle \approx 0.3 \mathrm{fm}$ [1]. This tentative consideration leads to $U_{p}=0 \mathrm{MeV}$ and $U_{n}=-1.6 \mathrm{MeV}$ or $B_{n}-B_{p}=1.6 \mathrm{MeV}$. The difference $B_{n}-B_{p}=2.0 \mathrm{MeV}$ contained in Eqs. (46) and (47) would lead to $\left\langle r_{i j}\right\rangle=0.24 \mathrm{fm}$ in reasonable agreement with the educated guess.

It may be expected that a calculation of the hadronic binding energy of the nucleon leads to interesting insights into the constituent-quark structure of the nucleon. At the present point of research we leave this as an open problem for further investigations.

\section{Hadron masses in the $\mathrm{SU}(3)$ sector}

In the $\mathrm{SU}(2)$ sector we have the $\pi$ mesons serving as Nambu-Goldstone boson and the $\sigma(666)$ meson serving as Higgs boson of strong interaction. In the $\mathrm{SU}(3)$ sector we expect an octet $\pi, K$ and $\eta$ of Nambu-Goldstone bosons and a nonet $\sigma(666), \kappa(800)$ and $f_{0}(980), a_{0}(980)$ of Higgs bosons of strong interaction. This latter case has been investigated in a previous paper [18]. Since the $\sigma(666)$ meson is given by the $|n \bar{n}\rangle=\mid(u \bar{u}+d \bar{d}\rangle / \sqrt{2}$ state one should expect that the $f_{0}(980)$ meson is given by the related $|s \bar{s}\rangle$ state. This, however cannot be the case because the mesons $f_{0}(980)$ and $a_{0}(980)$ have equal masses and, therefore, must have an equal fraction $f_{s}$ of strange quarks in the meson structure. There are arguments that the missing $|s \bar{s}\rangle$ scalar meson may be identified with the $f_{0}(1370)$ state. This has previously been pointed out by Hatsuda and Kunihiro [7] and recently by Fariborz et al. [19]. According to Hatsuda and Kunihiro the $f_{0}(1370)$ may be considered as a second sigma meson, $\sigma^{\prime}$, which takes over the rôle of the $\sigma$ meson when we replace $|n \bar{n}\rangle$ by $|s \bar{s}\rangle$. The mesons $\sigma$ and $\sigma^{\prime}$ differ by the fact that the wave function of the $\sigma$ meson contains two flavors, $N_{f}=2$, whereas the wave function of $\sigma^{\prime}$ contains only one flavor, $N_{f}=1$. This may lead to the consequence that the coupling constant of a $u$ or $d$ quark to the $\sigma$ meson may be different from the coupling strength of a $s$ quark to the $\sigma^{\prime}$ meson. This point will be investigated in the next subsection.

\subsection{Properties of the strange-quark $\sigma$ meson in the chiral limit}

The nonstrange and the strange-quark $\sigma$ mesons differ by the fact that we have flavor numbers $N_{f}=2$ for $\sigma$ and $N_{f}=1$ for $\sigma^{\prime}$. Due to this difference it may be expected that these two mesons have different quark-mesons coupling constants $g$ for the $u$ and $d$ quarks coupling to the $\sigma$ meson and the coupling constant $g_{S}$ for the of the $s$ quark to the $\sigma^{\prime}$ meson. This difference has been investigated by Delbourgo and Scadron [20,21] (see also [22]) on the basis of a diagrammatic approach. Using dimensional regularization the graphs sum up in the chiral limit to [21]

$$
\begin{aligned}
& \left(m_{\sigma}^{\mathrm{cl}}\right)^{2}=16 i N_{c} g^{2} \int \frac{d^{4} p}{(2 \pi)^{4}}\left[\frac{M^{2}}{\left(p^{2}-M^{2}\right)^{2}}-\frac{1}{p^{2}-M^{2}}\right]=\frac{N_{c} g^{2} M^{2}}{\pi^{2}} \\
& \left(m_{\sigma S}^{\mathrm{cl}}\right)^{2}=8 i N_{c} g_{S}^{2} \int \frac{d^{4} p}{(2 \pi)^{4}}\left[\frac{M_{S}^{2}}{\left(p^{2}-M_{S}^{2}\right)^{2}}-\frac{1}{p^{2}-M_{S}^{2}}\right]=\frac{N_{c} g_{S}^{2} M_{S}^{2}}{2 \pi^{2}} .
\end{aligned}
$$


Here use has been made of a $\Gamma$ function identity $\Gamma(2-l)+\Gamma(1-l) \rightarrow-1$ in $2 l=4$ dimensions. The conclusions drawn from these considerations are

$$
g=\frac{2 \pi}{\sqrt{3}}, \quad g_{S}=\sqrt{2} g, \quad m_{S}^{\mathrm{cl}}=2 M_{S} \text { and } M_{S}=\sqrt{2} M .
$$

The last of the relations in Eq. (51) implies that the vacuum expectation values of the nonstrange and strange-quark sigma fields are the same in the chiral limit. Keeping this in mind we arrive at

$$
M_{S}=461 \mathrm{MeV} \quad \text { and } \quad m_{\sigma S}^{\mathrm{cl}}=922 \mathrm{MeV} .
$$

\subsection{Current-quark mass of the $s$ quark}

In the $\mathrm{SU}(3)$ sector the effects of the Higgs boson enter into the mass generation process via the current-quark masses of the $u, d$ and $s$ quarks. In low-energy QCD the currentquark masses of the $u$ and $d$ quarks are well known to be $m_{u}^{0}=5 \mathrm{MeV}$ and $m_{d}^{0}=9 \mathrm{MeV}$, as already stated above. The current-quark mass of the $s$ quark is less well known and, therefore, requires some further investigation. Here we first make the attempt to exploit an analog of Eq. (20) given for $\pi$ meson and write down for the $K^{+}$

$$
m_{K^{+}}^{2}=\left(m_{u}^{0}+m_{s}^{0}\right) \frac{8 \pi}{\sqrt{3}} f_{K} .
$$

This equation allows to calculate the current-quark mass $m_{s}^{0}$ from the mass of the $K^{+}$ meson, the $K^{+}$meson decay constant $f_{K}$ and the current-quark mass $m_{u}^{0}$. Then with $m_{K^{+}}=493,67 \mathrm{MeV}, f_{K}=110.45 \mathrm{MeV}, m_{u}^{0}=5 \mathrm{MeV}$ we arrive at $m_{s}^{0}=147 \mathrm{MeV}$.

For the case of low-energy QCD the following values may be found in the literature: $m_{s}^{0}=(161 \pm 28) \mathrm{MeV}$ [7,23], $m_{s}^{0}=(175 \pm 55) \mathrm{MeV}[24] m_{s}^{0}=(199 \pm 33) \mathrm{MeV}$ [7, 25]. These data span the range from $m_{s}^{0}=133$ to $m_{s}^{0}=232 \mathrm{MeV}$ with the value $m_{s}^{0}=147$ $\mathrm{MeV}$ following from Eq. (53) being close to the lower limit. The following considerations appear to be justified. EW interaction alone should lead to a definite value, $m_{s}^{0}(\mathrm{EW})$,

of the current-quark mass of the strange quark and deviations from this value may be due to an incomplete decompositions of the effects of EW and strong interaction. Eq. (53) contains effects of strong-interaction only due to the decay constant $f_{K}$ which is well determined experimentally. Furthermore, the relation Eq. (53) is well justified through its close similarity with the corresponding Eq. (201) derived and found valid for the $\pi$ meson. This consideration leads to the supposition that a value around $m_{s}^{0}=147 \mathrm{MeV}$ may be identified with $m_{s}^{0}(\mathrm{EW})$.

\subsection{The masses and structures of scalar mesons}

In the $\mathrm{SU}(3)$ sector it has become customary to distinguish between scalar mesons with masses below $1 \mathrm{GeV}$ and scalar mesons with masses above $1 \mathrm{GeV}$. The properties of the scalar mesons with masses below $1 \mathrm{GeV}$ have been investigated and described in a previous paper [18. There is a nonet of scalar mesons with a $(q \bar{q})^{2}$ tetraquark structurecomponent coupled to a $q \bar{q}$ component. The reason for the assumption of a tetraquark 
structure-component is that in a $q \bar{q}$ model the electrically neutral $a_{0}(980)$ meson should have a quark structure in the form $(-u \bar{u}+d \bar{d}) / \sqrt{2}$ and the $f_{0}(980)$ meson a quark structure in the form $s \bar{s}$. This would lead to the consequence that the masses should be different, whereas in reality they are equal to each other. On the other hand in a tetraquark model the fraction of strange quarks $f_{s}$ is equal in the two mesons as can be seen in Table 2 .

Table 2: Summary of scalar mesons in the $(q \bar{q})^{2}$ representation according to [26]. $Y$ : hypercharge, $I_{3}$ : isospin component, $f_{s}$ : fraction of strange and/or anti-strange quarks in the tetraquark structure.

\begin{tabular}{|c|c|c|c|c|c|c|c|}
\hline$Y / I_{3}$ & -1 & $-1 / 2$ & 0 & $+1 / 2$ & +1 & Meson & $f_{s}$ \\
\hline+1 & & $d \bar{s} u \bar{u}$ & & $u \bar{s} d \bar{d}$ & & $\kappa(800)$ & $1 / 4$ \\
\hline 0 & & & $u \bar{d} d \bar{u}$ & & & $\sigma(600)$ & 0 \\
\hline 0 & $d \bar{u} s \bar{s}$ & & $s \bar{s}(u \bar{u}-d \bar{d}) / \sqrt{2}$ & & $u \bar{d} s \bar{s}$ & $a_{0}(980)$ & $1 / 2$ \\
\hline 0 & & & $s \bar{s}(u \bar{u}+d \bar{d}) / \sqrt{2}$ & & & $f_{0}(980)$ & $1 / 2$ \\
\hline-1 & & $s \bar{u} d \bar{d}$ & & $s \bar{d} u \bar{u}$ & & $\kappa(800)$ & $1 / 4$ \\
\hline
\end{tabular}

The tetraquark structure of the $\sigma(600)$ meson needs a special consideration. For this purpose we study the two reaction chains given in Eqs. (54) and (55)

$$
\begin{aligned}
& \gamma \gamma \rightarrow(u \bar{u}+d \bar{d}) / \sqrt{2} \rightarrow u \bar{d} d \bar{u} \rightarrow \pi \pi \\
& \gamma \gamma \rightarrow(u \bar{u}+d \bar{d}) / \sqrt{2} \rightarrow N \bar{N} .
\end{aligned}
$$

Eq. (54) describes the two-photon production of a pion pair. The two photons first excite the $q \bar{q}$ structure component of the $\sigma$ meson which is simpler than the tetraquark structure component and therefore has a larger transition matrix element. Thereafter a rearrangement of the structure leads to the tetraquark structure which then decays into two pions. In this reaction chain the $q \bar{q}$ structure component serves as a doorway state for the two-photon excitation of the tetraquark structure component [18] . Eq. (55) describes Compton scattering via the $t$-channel. In this case only the $q \bar{q}$ structure plays a rôle. For kinematical reasons the $\sigma$ meson described in Eq. (55) shows up as a narrow resonance having a definite mass of $m_{\sigma}=666 \mathrm{MeV}$, whereas the $\sigma$ meson described in Eq. (54) corresponds to a pole on the second Riemann sheet.

It is apparent that Table 2 does not contain a scalar meson having a $s \bar{s}$ structure. This leads to the expectation that one of the scalar mesons located above $1 \mathrm{GeV}$ should have this structure. This expectation has been confirmed by Hatsuda and Kunihiro [7] who applied RPA techniques to the mass relation of the NJL model. In this way it has been predicted that two $\sigma$ mesons exist, viz.

$$
m_{\sigma}=668.0 \mathrm{MeV} \text {, and } m_{\sigma^{\prime}}=1344 \mathrm{MeV}
$$

where $|\sigma\rangle=|(u \bar{u}+d \bar{d}) / \sqrt{2}\rangle$ and $\left|\sigma^{\prime}\right\rangle=|s \bar{s}\rangle$. The mass of the $\sigma$ meson is in close agreement with the mass $m_{\sigma}=666 \mathrm{MeV}$ derived above, showing that our method and the one of Hatsuda and Kunihiro [7] are essentially equivalent. This gives us confidence that it is 
appropriate to use $m_{\sigma^{\prime}}=1344 \mathrm{MeV}$ as one basis for predictions of masses of scalar mesons containing strange quarks, in case the effects of the current quarks are included. The other basis is the predicted constituent-quark mass in the chiral limit given in Eq. (52), i.e. for the case that the effects of the current quarks are not included. In the following we discuss three models for the mass generation of scalar mesons differing by the procedure of combining the effects strong-interaction and EW interaction. These models are extensions of the corresponding models used in the $\mathrm{SU}(2)$ sector.

\subsubsection{First overview on the masses of scalar mesons without and with includ- ing the effects EW interaction}

The masses of scalar mesons may be composed of the masses of the two $\sigma$ mesons making contributions in proportion to the fraction of non-strange quarks and strange quarks, respectively. The appropriate mass formulae are

$$
\begin{aligned}
& m_{\mathrm{scalar}}^{\mathrm{cl}}=\left(1-f_{s}\right) m_{\sigma}^{\mathrm{cl}}+f_{s} m_{\sigma^{\prime}}^{\mathrm{cl}}, \\
& m_{\mathrm{scalar}}=\left(1-f_{s}\right) m_{\sigma}+f_{s} m_{\sigma^{\prime}}
\end{aligned}
$$

where Eq. (57) refers to the chiral limit where the effects of EW interaction are disregarded and Eq. (58) to the case where these effects are included. Eq. (57) is evaluated using $m_{\sigma}^{\mathrm{cl}}=652 \mathrm{MeV}$ and $m_{\sigma^{\prime}}^{\mathrm{cl}}=922 \mathrm{MeV}$ as predicted in section 4.1. Eq.(58) is evaluated using the standard value of the mass of the $\sigma$ meson $m_{\sigma}=666 \mathrm{MeV}$ and $m_{\sigma^{\prime}}=1344$ $\mathrm{MeV}$ as predicted by Hatsuda and Kunihiro [7]. The results of these mass predictions are given in columns 4, (a) and 5, (b) of Table 3, By comparing the masses in column (a) with those of column (b) we see that the largest part of the mass is already present in the chiral limit, i.e. before EW interaction is taken into account. The values in column (b) have to be compared with the experimental values and show a reasonable agreement. These experimental values given in Table 3 have been obtained as follows.

Table 3: Decuplet of scalar mesons including $f_{0}(1370) \equiv \sigma^{\prime}(1344)$. (a) Scalar-meson masses in the chiral limit according to Eq. (57), (b) Scalar-meson masses including the effects of EW interaction according to Eq. (58), (c) Scalar-meson masses including the effects EW interaction as predicted through the masses of pseudo-Goldstone bosons according to Eqs. (60), (61), (d) Current-quark masses $m_{s}^{0}$ of the strange quark obtained through adjustments to experimental data according to Eq. (62).

\begin{tabular}{|l|ll|l|l|l|l|l|}
\hline meson & exp. $m_{\text {scalar }}$ & ref. & $f_{s}$ & $m_{\text {scalar }}^{\text {cl }}(\mathrm{a})$ & $m_{\text {scalar }}(\mathrm{b})$ & $m_{\text {scalar }}(\mathrm{c})$ & $m_{s}^{0}(\mathrm{~d})$ \\
\hline$\sigma(600)$ & $600 \pm 70$ & {$[14$} & 0 & 652 & 666 & 666 & - \\
$\kappa(800)$ & $700-900$ & {$[27$} & $1 / 4$ & 720 & 836 & 806 & 139 \\
$f_{0}(980)$, & $980 \pm 20$ & {$[27$} & $1 / 2$ & 787 & 1005 & 929 & 191 \\
$a_{0}(980)$ & $990 \pm 20$ & {$[27$} & $1 / 2$ & 787 & 1005 & 929 & 191 \\
$f_{0}(1370)$ & $1368 \pm 22$ & {$[28$} & 1 & 922 & 1344 & - & 244 \\
\hline
\end{tabular}

The experimental masses of the $\kappa(800), a_{0}(980)$ and $f_{0}(980)$ mesons are taken from the Particle Data Group [27]. The mass of the $f_{0}(1370)$ meson has been determined in three 
experiments via the $\bar{p} p \rightarrow 5 \pi$ reaction [28], leading to the weighted average listed in line 6 of column 2 in Table 3. It is straightforward to identify the predicted scalar meson $\sigma^{\prime}(1344)$ with the observed scalar meson $f_{0}(1370)$. One argument for this identification is the agreement of the values obtained for the masses. An other argument is that $f_{0}(980)$ is excluded because of its tetraquark structure. A third argument is based on calculations of Fariborz et al. [19] leading to arguments in favor of a $s \bar{s}$ structure. One consequence of these findings is that the constituent mass of the $s$ quark including the effects of $\mathrm{EW}$ interaction is rather large, viz.

$$
m_{s}=\frac{1}{2} \times 1344 \mathrm{MeV}=672 \mathrm{MeV} .
$$

\subsubsection{Effects of EW mass generation calculated via the masses of pseudo- Goldstone bosons}

In the preceeding subsection the masses of scalar mesons are constructed for the two cases where the effects of EW interaction are not included (column (a)) and included (column (b)). The considerations presented have the advantage that the effect of EW interaction on the mass of the scalar meson are clearly demonstrated and quantitative values of the masses for the two cases are predicted.

A further independent method to take into account the effects of EW interaction may be obtained from the supposition that the masses of the scalar mesons $\kappa(800)$ and $f_{0}(980), a_{0}(980)$ may be calculated using mass formulae analogous to Eq. (22) which was written down for the $\sigma$ meson. These formulae are

$$
\begin{aligned}
& m_{\kappa}=\left(\left(m_{\mathrm{scalar}}^{\mathrm{cl}}(\kappa)\right)^{2}+\frac{1}{2}\left(m_{\pi}^{2}+m_{K}^{2}\right)\right)^{1 / 2} \\
& m_{f_{0}, a_{0}}=\left(\left(m_{\mathrm{scalar}}^{\mathrm{cl}}\left(f_{0}, a_{0}\right)\right)^{2}+\left(m_{K}\right)^{2}\right)^{1 / 2} .
\end{aligned}
$$

In Eqs. (60) and (61) use is made of the masses of pseudoscalar mesons $\pi$ and $K$ in order to take into account the effects of the EW interaction. These mesons are pseudoGoldstone bosons and, therefore, have the property that their masses tend to zero in the chiral limit, as requested in Eqs. (60) and (61). The results of this calculation are listed in column 6 (c) of Table 3. The agreement of the two methods of calculation given in column (b) and (c) of Table 3 is good for the $\kappa(800)$ meson but shows the tendency of a deviation in case of the $f_{0}(980)$ and $a_{0}(980)$ mesons. This deviation can be removed by replacing the K-meson mass by larger value of $590 \mathrm{MeV}$, showing that Eq. (61) is a reasonable but not perfect approximation.

\subsubsection{Effects of EW interaction taken in account by adding current-quark masses to the $\sigma^{\prime}$ meson mass in the chiral limit}

A further option for the calculation of the EW-interaction part of scalar meson masses may be obtained from the ansatz

$$
m_{\sigma^{\prime}}=m_{\sigma^{\prime}}^{\mathrm{cl}}+2 m_{s}^{0}
$$


where $m_{\sigma^{\prime}}^{\mathrm{cl}}=922 \mathrm{MeV}$, as shown in line 6 of column (a) in Table 3. This ansatz is an extension of the relation $m_{\sigma}=m_{\sigma}^{c l}+\left(m_{u}^{0}+m_{d}^{0}\right)$ derived in Eq. (25). Here the currentquark mass, $m_{s}^{0}$, is an adjustable parameter determined by adjusting the predicted mass according to Eq. (58) to the experimental scalar mass.

The result of this adjustment procedure is shown in column $(\mathrm{d})$ of Table 3. For the meson $\kappa(800)$ the result $m_{s}^{0}=136 \mathrm{MeV}$ is in good agreement with the the result $m_{s}^{0}=147$ $\mathrm{MeV}$ obtained from the mass of $K$ meson (see subsection 4.2). At larger masses of the scalar mesons there is a drastic increase of the adjusted current-quark mass $m_{s}^{0}$, but the results obtained remain in the range $m_{s}^{0}=133$ to $232 \mathrm{MeV}$ of values found in previous investigations (see subsection 4.2).

From this finding the following conclusions may be drawn. First of all there should be a definite value for the current-quark mass of the strange quark, viz. $m_{s}^{0}(\mathrm{EW})$, which is the result of a genuine EW mass production process related to the Higgs boson. The different model dependent values $m_{s}^{0}$ obtained by adjusting to experimental data contain additional contributions from the $\langle q \bar{q}\rangle$ vacuum condensate which is not taken care of by the scalar mass calculated for the chiral limit. These additional contributions may be quite sizable as shown by the increase of the values in column (d) of Table 3 with increasing mass of the scalar meson. An explanation of this additional contribution would be an interesting topic for further investigations.

\subsection{The masses of octet baryons}

Scalar mesons and baryons differ by the fact that scalar mesons are loosely bound so that the mesons mass can be identified with the sum of constituent-quark masses, whereas in the case of baryon masses binding energies have to be taken into account. In Table 4 a test of this concept is carried out. In column (a) the average experimental masses of the three groups of octet baryons are shown. In columns (b) and (c) the corresponding predicted masses in the chiral limit and including the effects of EW interaction are listed. Apparently these predicted masses of column (c) are larger than the experimental ones,

Table 4: (a): average experimental masses of the three groups of baryons. (b): sum of the masses of the three constituent quarks in the chiral limit. (c): sum of the masses of the three constituent quarks including the effects of EW interaction. (d) binding energy (B) per number of quarks $(\mathrm{A}=3$ ) (in units of $\mathrm{MeV}$ ).

\begin{tabular}{|l|l|l|l|l|}
\hline baryon & $m_{\text {exp. }}(\mathrm{a})$ & $m_{\text {theor. }}^{\text {cl }}(\mathrm{b})$ & $m_{\text {theor. }}(\mathrm{c})$ & $\mathrm{B} / \mathrm{A}(\mathrm{d})$ \\
\hline$p, n$ & 939 & 978 & 999 & 20 \\
$\Lambda, \Sigma^{ \pm, 0}$ & 1234 & 1113 & 1338 & 55 \\
$\Xi^{0,-}$ & 1318 & 1248 & 1677 & 120 \\
\hline
\end{tabular}

confirming the expectation that binding energies are involved in the formation of the total baryon mass. The binding energies $B$ per number $A=3$ of constituent quarks are listed in column (d). These binding energies increase with the fraction of strange quarks located in the baryon. This interesting finding needs an explanation. 


\subsection{The magnetic moments of Hyperons}

The magnetic moments of hyperons have been predicted making use of different assumptions about the masses of the constituent quarks (see e.g. [29]). Within the quark model the masses of the constituent quarks are the only parameters in the prediction of magnetic moments. Therefore, within this model information on the constituent-quark masses is obtained by comparing predicted magnetic moments with experimental values.

In the present investigation we use $m_{u}=331 \mathrm{MeV}, m_{d}=335 \mathrm{MeV}$ and $m_{s}=672$ $\mathrm{MeV}$. With these constituent-quark masses the predicted magnetic moments $\mu^{\text {theor. }}$ given

Table 5: Constituent mass of strange quark calculated from magnetic moments

\begin{tabular}{|l|c|l|r|}
\hline hyperon & $\left(9 / m_{p}\right) \times \mu^{\text {theor. }}$ & $\mu_{\text {exp. }}$ & $\mu^{\text {theor. }}$ \\
\hline$\Lambda$ & $-3 / m_{s}$ & $-0.613 \pm 0.004$ & -0.465 \\
$\Sigma^{+}$ & $8 / m_{u}+1 / m_{s}$ & $2.458 \pm 0.010$ & 2.675 \\
$\Sigma^{-}$ & $-4 / m_{d}+1 / m_{s}$ & $-1.160 \pm 0.025$ & -1.090 \\
$\Xi^{0}$ & $-4 / m_{s}-2 / m_{u}$ & $-1.250 \pm 0.014$ & -1.250 \\
$\Xi^{-}$ & $-4 / m_{s}+1 / m_{d}$ & $-0.6507 \pm 0.0025$ & -0.309 \\
$\Omega^{-}$ & $-9 / m_{s}$ & $-2.02 \pm 0.05$ & -1.396 \\
\hline
\end{tabular}

in column 4 of Table 5 are obtained. The value predicted for the $\Xi^{0}$ hyperon is in perfect agreement with experimental value. In other cases the agreement is less pronounced. However, by using a different choice for the constituent mass of the strange quark no improvement of the general agreement is obtained. The conclusion we have to draw is that our set of constituent-quark masses is in line with the experimental magnetic moments, though there is no strong support for the specific value adopted for the $s$ quark. The information obtained from the masses of scalar mesons on the constituent mass of the $s$ quark appears to be more reliable than the one obtained from magnetic moments.

\section{$5 \quad$ Summary and Conclusions}

In the forgoing it has been shown that the prediction of scalar-meson and baryon masses can be extended from the $\mathrm{SU}(2)$ sector to the $\mathrm{SU}(3)$ sector by making use of a second $\sigma$ meson, viz. $\sigma^{\prime}(1344)$, having the $|s \bar{s}\rangle$ structure. This second $\sigma$ meson implies that the constituent-quark mass of the $s$ quark when including the effects of the Higgs boson is $m_{s}=672 \mathrm{MeV}$. This large constituent-quark mass leads to reasonable predictions of the masses of scalar mesons below $1 \mathrm{GeV}$ and of the masses of octet baryons. Furthermore, also the magnetic moments of octet baryons are predicted leading to results being in line with the experimental values.

The masses of constituent quarks are composed of the masses $M_{q}$ predicted for the chiral limit and the mass of the respective current quark $m_{q}^{0}$ provided by the Higgs boson (EW interaction) alone. For scalar mesons the sum of $M_{q}$ and $m_{q}^{0}$ leads to a zero-order approximation for the constituent-quark mass $m_{q}$, but there are dynamical effects described by the NJL model which modify the simple relation $m_{q}=M_{q}+m_{q}^{0}$, except for 


\section{$\operatorname{Mass}[\mathrm{GeV}]$}

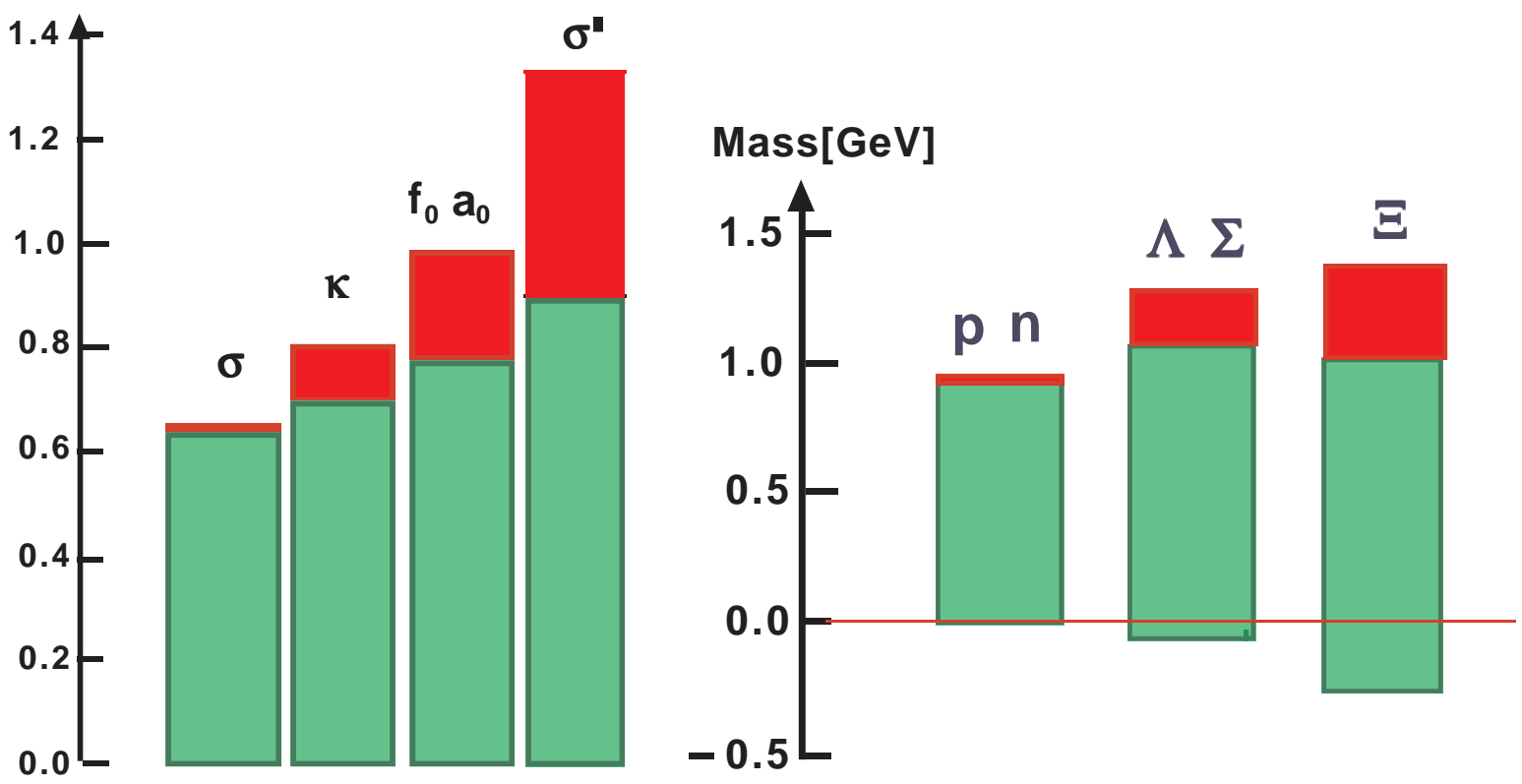

Figure 2: Masses generated via strong-interaction (green or light-grey boxes) and EW interaction (red or dark-grey boxes). Left panel: Scalar mesons. Right panel: Octet baryons. The negative parts of the green boxes in the right panel correspond the binding energies observed in the octet baryons. Without the effects of the Higgs boson only the masses represented by the green boxes would be present. The red boxes correspond to the effects of the Higgs boson.

the non-strange sector where this relation is a good approximation. Similar results are obtained for the octet baryons. A difference between the scalar mesons and the octet baryons is that that for scalar mesons binding energies do not play a rôle whereas they are of importance in case of octet baryons.

In Figure 2 a graphical a representation of mass generation is given. The green boxes correspond to masses in a world without the Higgs boson, whereas the red boxes represent the effects of the Higgs boson on the mass generation process. 


\section{References}

[1] M. Schumacher, Ann. Phys. (Berlin) 526, 215 (2014), arXiv:1403.7804 [hep-ph].

[2] Y. Nambu, G. Jona-Lasinio, Phys. Rev 122, 345 (1961); 124, 246 (1961).

[3] D. Lurié, A.J. MacFarlane, Phys. Rev. B 136, 816 (1964).

[4] T. Eguchi, Phys. Rev. D 14, 2755 (1976); 17, 611 (1978).

[5] U. Vogl, W. Weise, Prog. Part.Nucl. Phys. 27, 195 (1991).

[6] S.P. Klevansky, Rev. Mod. Phys. 64, 649 (1992).

[7] T. Hatsuda, T. Kunihiro, Physics Reports 247, 221 (1994).

[8] J. Bijnens, Physics Reports 265, 369 (1996).

[9] M. Gell-Mann, M. Levy, Nuovo. Cim 16, 705 (1960);

V. De Alfaro, S. Fubini, G. Furlan, C. Rosetti, Currents in Hadron Physics, North Holland, Amsterdam 1973.

[10] J. Schwinger, Ann Phys. (NY) 2, 407 (1957).

[11] F. Englert, Ann. Phys. (Berlin) 526, 201 (2014).

[12] P.W. Higgs, Ann. Phys. (Berlin) 526, 211 (2014).

[13] F. Halzen, A.D. Martin, Quarks and Leptons: An Introductory Course in Modern Particle Physics, JOHN WILEY \& SONS, New York 1984.

[14] M. Schumacher, M.D. Scadron, Fortschr. Phys. 61, 703 (2013), arXiv:1301.1567 [hep$\mathrm{ph}$.

[15] G. Galler et al., Phys. Lett. B 503, 245 (2001).

[16] S. Wolf et al., Eur. Phys. J. A 12, 231 (2001).

[17] M. Schumacher, Eur. Phys. J. C 67, 283 (2010), arXiv:1001.0500 [hep-ph].

[18] M. Schumacher, J. Phys. G: Nucl. Part. Phys. 38, 083001 (2011), arXiv:1106.1015 [hep-ph].

[19] A. H. Fariborz, A. Azizi, A. Asrar, arXiv:1503.05041 [hep-ph].

[20] R. Delbourgo, M. Scadron, Mod. Phys. Lett. A 10, 251 (1995), arXiv:hep-ph/9910242 R. Delbourgo, M. D. Scadron, A.A. Rawlinson, Mod. Phys. Lett. A 13, 1893 (1998), arXiv:hep-ph/980750.

[21] R. Delbourgo, M. Scadron, Int. J. Mod. Phys. A 13, 657 (1998), arXiv:hep-ph/9807504.

[22] M. Schumacher, Eur. Phys. J. A 30, 413 (2006); A 32, 121 (2007) (E), arXiv:hep-ph/0609040. 
[23] S. Narison, Riv. Nuovo Cimento 10 (1987) No. 2

[24] J. Gasser, H. Leutwyler, Physics Reports 87, 77 (1982).

[25] C. A. Dominguez, E. De Rafael, Ann. Phys. 174, 372 (1987).

[26] R.J. Jaffe, Phys. Rev. D 15, 267 (1977); D 15, 281 (1977); D 17, 1444 (1978).

[27] K.A. Olive et al. (Particle Data Group) Chin. Phys. C 38, 090001 (2014).

[28] E. Klempt, Nucl. Phys. A 629, 131c (1998).

[29] M.D. Scadron, R. Delbourgo, R. Rupp, J. Phys. G 32, 735 (2006), arXiv:hep-ph/0603196. 